\title{
Thinness and Obesity: A Model of Food Consumption, Health Concerns, and Social Pressure*
}

\author{
Davide Dragone ${ }^{\dagger} \quad$ Luca Savorelli ${ }^{\ddagger}$
}

The Suntory Centre

Suntory and Toyota International Centres for

Economics and Related Disciplines

London School of Economics and Political Science

Houghton Street

London WC2A 2AE

EOPP/2010/17

Tel: (020) 79556674

\footnotetext{
* We thank Oriana Bandiera, Francesca Barigozzi, Maria Bigoni, Marco Casari, Arsen Palestini, Paolo Vanin, and the audience of the Public Economics UK Workshop at the IFS in London, the Max Planck Institute of Economics in Jena, the Department of Economics in Konstanz, and the Departments of Economics in Bologna for their useful comments and suggestions. Luca Savorelli is grateful to STICERD - The London School of Economics and Political Science, where part of this work has been developed while he was a visiting research student. The usual disclaimer applies.

† Dipartimento di Scienze Economiche, Università di Bologna, Piazza Scaravilli 2, 40126, Bologna, Italia; e-mail: davide.dragone@unibo.it.

‡ Dipartimento di Scienze Economiche, Università di Bologna, Piazza Scaravilli 2, 40126 Bologna, Italia; and Dipartimento di Economia Politica, Università di Siena, Piazza S. Francesco 7, 53100, Siena, Italia; e-mail: 1.savorelli@Ise.ac.uk.
} 


\begin{abstract}
The increasing concern of the policy maker about eating behavior has focused on the spread of obesity and on the evidence of a consistent number of individuals dieting despite being underweight. As the latter behavior is often attributed to the social pressure to be thin, some governments have already taken actions to ban ultra-thin ideals and testimonials. Assuming that people are heterogeneous in their healthy weights, but are exposed to the same ideal body weight, this paper proposes a theoretical framework to assess whether increasing the ideal body weight is socially desirable, both from a welfare and from a health point of view. If being overweight is the average condition and the ideal body weight is thin, increasing the ideal body weight may increase welfare by reducing social pressure. By contrast, health is on average reduced, since people depart even further from their healthy weight. Given that in the US and in Europe people are on average overweight, we conclude that these policies, even when are welfare improving, may foster the obesity epidemic.
\end{abstract}

JEL classification: D91, I18.

Keywords: Body Weight, Diet, Obesity, Social Pressure, Underweight. 
This series is published by the Economic Organisation and Public Policy Programme (EOPP) located within the Suntory and Toyota International Centres for Economics and Related Disciplines (STICERD) at the London School of Economics and Political Science. This new series is an amalgamation of the Development Economics Discussion Papers and the Political Economy and Public Policy Discussion Papers. The programme was established in October 1998 as a successor to the Development Economics Research Programme. The work of the programme is mainly in the fields of development economics, public economics and political economy. It is directed by Maitreesh Ghatak. Oriana Bandiera, Robin Burgess, and Andrea Prat serve as codirectors, and associated faculty consist of Timothy Besley, Jean-Paul Faguet, Henrik Kleven, Valentino Larcinese, Gerard Padro i Miquel, Torsten Persson, Nicholas Stern, and Daniel M. Sturm. Further details about the programme and its work can be viewed on our web site at http://sticerd.lse.ac.uk/research/eopp.

Our Discussion Paper series is available to download at:

http://sticerd.lse.ac.uk/_new/publications/series.asp?prog=EOPP

For any other information relating to this series please contact Leila Alberici on:

Telephone: UK+20 79556674

Fax: $\quad$ UK+20 $\quad$ : $\quad$ (a556951

Email: $\quad$ l.alberici @lse.ac.uk

(C) The authors. All rights reserved. Short sections of text, not to exceed two paragraphs, may be quoted without explicit permission provided that full credit, including (C) notice, is given to the source. 


\section{Introduction}

The governments of Italy, Germany and Spain have recently signed agreements with the fashion business associations to set in detail some aspects of the industry productive decisions, communication strategies, and labelling of products. The agreements favour, for example, increases in the production of large-sized clothes, in the minimum size of models on the catwalks, and of mannequins in the shops; or they forbid the denomination "fat size" for size 46. The explicit goal of these policies is to reduce unhealthy eating behavior among young people, in particular young women, by affecting the ideal of beauty that the media and the fashion industry disseminate 1 . This paper proposes a theoretical framework to assess whether increasing the ideal body weight is socially desirable, both from a welfare and from a health point of view.

Given the paramount relevance of Europe in the international fashion market, these agreements can have important world-wide consequences on the fashion industry, a global business that in the U.S. has a turnover in excess of $\$ 200$ billion ${ }^{2}$. Another consequence, on which we focus in this paper, is the effect that these policies may have on people's eating behavior, health and welfare. When reading the content of the agreements, it is clear that both the government and the fashion industry agree that fashion is a powerful trend-setter. It not only influences what clothes, styles and colors are trendy, but also defines how a person should appear to be desirable. This includes body shape and body weight. People often recognize that being attractive according to the yardsticks set by the media and the fashion industry is out of reach. It is also true that not everybody judges herself or himself according to these ideal body shapes and weights. There is however evidence that plain-looking people tend to find worse jobs and receive lower wages (Hamermesh and Biddle, 1994) and, with reference to body weight, that being overweight determines multiple forms of prejudice and discrimination (Cawley, 2004; Morris, 2006; Puhl and Heuer, 2009). In other words, there is evidence that those not conforming to the ideal body weight, are stigmatized and socially sanctioned (Strahan et al., 2006).

In this paper we formalize these arguments considering how a given ideal body weight affects eating behavior, and consequently body weight and health. We propose a dynamic model of individual eating behavior where a forward looking agent chooses how much food to consume. Utility depends on food consumption and on body weight, the latter being endogenously determined by the difference between calories intake and expenditure. If the agent's body weight is either higher or lower than her healthy weight, the agent suffers a reduction in her health. The agent also suffers a disutility cost when her body weight does not conform to an ideal weight

\footnotetext{
${ }^{1}$ Along the same reasoning, in 2008 France approved a law that makes the promotion of extreme forms of slenderness a criminal offense.

${ }^{2}$ The apparel industry's annual sales in the U.S. are more than the sum of the sales of books, movies and music. In New York, one of the fashion capitals, the fashion industry is the third-employer, the first two being finance and health-care; see Hemphill and Suk (2009), Raustiala and Sprigman (2009).
} 
that is exogenously determined by the society, and that cannot be influenced by the agent. Since the healthy weight and the ideal weight usually do not coincide, agents have to trade-off the health and social consequences of their food intake. This results in a variety of eating behaviors, including the possibility of optimally choosing to be on diet despite being underweight.

Under the assumption that the mentioned policies are effective in increasing the ideal body weight, we provide a normative assessment of their desirability, both in terms of aggregate utility and aggregate health. The distinction is relevant because a welfare-maximizing policy does not coincide with a health-maximizing one. Assuming that people are heterogeneous in their healthy weights, but are exposed to the same ideal body weight, we show that increasing the ideal body weight induces people to increase their food intake and their body weight. If people are underweight and stay on a diet, increasing the ideal body weight allows both aggregate welfare and health to be improved. If people are overweight and on a diet, however, increasing the ideal body weight can improve overall utility, but it worsens health because it induces people to become even more overweight. A precise assessment of the relevant policy objective and of who will be influenced by the policy is therefore critical for determining the desirability of an increase in the ideal body weight. Given that in the US and in Europe people are on average overweight, these policies, even when are welfare improving, may thus foster the obesity epidemic.

Our approach is in line with Akerlof and Kranton (2000), who consider the fact that individuals belong to social categories, and that each category can be associated with different ideal physical attributes and prescribed behaviors. The existence of these references generates a pressure to conform to the ideal attribute or behavior, and deviations from them are socially sanctioned 3 Akerlof and Kranton observe that public policies sometimes are directed at manipulating these references, and consequently individual behavior. A notable example is the stigmatization of smokers and the restrictions on smoking advertising aimed at cutting the consumption of tobacco, which have been effective at reducing smoking in the population (Akerlof and Kranton, 2000; Philipson and Posner, 2008). Analogously, in this paper we provide a theoretical evaluation of the welfare and health effects of policies aimed at affecting the social desirability of body weight, with the goal of counteracting the "slimming mania" of young people.

Our paper contributes to the sparse economic literature on eating behavior in three directions. First, we extend the previous literature on the "obesity epidemics" (Philipson and Posner, 1999;

\footnotetext{
${ }^{3}$ Akerlof and Kranton (2000) introduce the concept of social norm to study prescribed behaviors (i.e. people's views of how they, and others, should or should not behave), as well as prescribed attributes. According to the second part of the definition, we could refer to the existence of social pressure on body weight as a sociocultural norm of appearence (as in Levy, 2002). There exist alternative definitions for social norms. For example, Bicchieri (2006) defines a social norm in terms of social expectations, while Fehr and Gächter (2000) refer to it as a behavioral regularity. Additionally, norms can be both externally and internally enforced by social pressure and individual feelings of guilt, embarassment, and anxiety (Elster, 1989). We do not use the term social norm to avoid confusion, and we refer to the social cost of not conforming to the ideal body weight.
} 
Levy, 2002; Yaniv, 2002; Dragone, 2009; Yaniv et al., 2009) by showing that being underweight can be the rational outcome of a maximizing agent. Second, we show that a single model with social pressure can produce a variety of empirically observed eating behaviors. Finally, we contribute to the small body of papers that focus on policies aimed at affecting eating behavior, such as the discussion on the role of fat taxes and thin subsidies (Yaniv et al., 2009), the implementation of educational programs (Acs and Lyles, 2007; Philipson and Posner, 2008), and the enhancement of access to information (Downs et al., 2009; Wansink et al., 2009). To the best of our knowledge, laws and agreements aimed at regulating eating behavior by affecting the ideal body weight have not been discussed before.

The paper is structured as follows. In the following section we will present in detail the content of the existing agreements and the underlying rationale. In the third section we will develop the model of individual food consumption and endogenous body weight. In the fourth section we will study the impact of an increase in the ideal body weight on both welfare and health, and we will discuss the implications of the existing policies. Finally, in the last section we will provide conclusions and directions for future research.

\section{2 "Together against slimming mania"}

On 23 January 2006, the Italian Ministry for Youth and Sports Activities signed an agreement with the fashion industry to prohibit the participation of anorexic models in the fashion shows, to increase the production of clothes sized 46-48, and to avoid promoting slender ideals. After one year, on 23 January 2007, the Spanish Ministry of Health and the Spanish fashion industry and stylists associations signed a similar agreement where they establish that models and mannequins should resemble the population biometrics. They set a minimum size for models and mannequins, a minimum body mass index (BMI) for participants to beauty contests, and they forbid the denomination "fat size" for clothes sized 46. France opted for a tougher remedy. To counteract the proliferation of websites promoting anorexia as a lifestyle (the so-called "ProAna" movement), from 15 April 2008 those who explicitly promote extreme forms of slenderness can be punished with a 30.000 Euro fine and two years prison 4 . After a few months, on 11 July 2008, the German Ministry of Health and the German textile and fashion industry also signed a national charter. Significantly titled "Life Carries Weight - Together against slimming mania", the goal of the charter is "to initiate a process of reorientation among children, young people and adults regarding prevailing beauty ideals" by promoting and propagating "a healthy body im-

\footnotetext{
${ }^{4}$ Anorexia nervosa is an eating disorder that can originate from multiple causes. Cultural factors, such as the promotion of thinness as the ideal female form in Western industrialized nations, have been recognized as one of the possible causes. People in professions where there is a particular social pressure to be thin (such as models and dancers) were much more likely to develop anorexia nervosa during the course of their career (Garner and Garfinkel, 2009, among others).
} 
age" and "unequivocally reject[ing] the unhealthy ideal of extreme thinness particularly among girls and women". It also sets a minimum BMI for fashion models and forbids the publication of images or photos of extremely thin models. Finally, it proposes to promote a discussion at European level aimed at writing a European Charter to channel the individual national efforts.

The above polices are based on the assumption that the fashion industry and the media have a major role in disseminating references on the ideal body weight to achieve. This assumption, that is clearly stated in the German Charter 5 , is corroborated by the psychological and sociological literature (e.g. Garner et al., 1980, 2009; Fallon, 1990; Wolf, 1991; Kilbourne, 1994; Smolak, 1996; Swami, 2006, and Ahern et al., 2008). According to this literature, since the 1960s in Western industrialized countries attractiveness has been represented by a thin, fit body shape for females and a lean, muscular physique for males. Whether these ideal body weights influence people's eating behavior and whether the fashion industry is responsible for people's improper eating behavior has been a matter of debate. On one hand, some observe that not everybody judges herself or himself according to the ideal weight proposed by the media, especially if one sees them as unrealistic and unattainable. According to this perspective, the fashion industry and the media should freely choose their marketing strategies and their testimonials, and no external intervention is needed ${ }^{6}$ On the other hand, some underline that, even if a person does not individually care about the prevalent ideal body weight, it can turn out to be relevant if other people (say, a potential employer or partner) discriminate against those who are overweight or underweight. Thus, while the fashion industry and the media make their profit-maximizing choices, they also produce an externality: the social pressure to conform to a given ideal body weight. From this perspective, the existence of a social pressure that sanctions those who do not conform to the ideal body weight can affect the optimal individual eating behavior. More specifically, the slender ideal is considered to induce improper eating behavior, negative consequences on people's health, and ultimately well-being, which has motivated the governments of France, Italy, Germany and Spain and the national fashion associations to agree on forbidding the promotion of ultra-thin women and increasing the ideal body weight.7

In the following section we include the latter argument in a model of individual eating behavior. This allows us to study the effect of social pressure to conform to an ideal, exogenous

\footnotetext{
${ }^{5}$ The German charter says "even if the number of super-thin models employed in Germany is far lower than in other European countries, the [fashion] industry still exerts a tremendous influence through the media".

${ }^{6}$ As an example, see the view point taken by Armstrong and Turner on The Times, Sept. 23, 2006. Explaining why the slender ideal has been promoted by the fashion industry in the last 50 years is out the scope of this paper. As economists, it is natural to think that the marketing choices we observe are driven by profit-maximizing strategies. For what concerns the use of ultra-thin models, this seems to be the case as most designers clearly state that clothes hang better on thin fashion models.

${ }^{7}$ Not all the governments and fashion industry associations have agreed on undertaking this kind of measure. For example, since 2006 the British Fashion Council has decided not to ban very thin models from the London Fashion Weeks. Interestingly, some British stylists independently decided to promote a healthier ideal of beauty.
} 
body weight on individual food intake and body weight, and to provide a guideline to evaluate the above mentioned policies.

\section{The model}

Consider a scenario where the population of a given country is made up of distinct groups, indexed by $G \in\{1,2, \ldots, M\}$. In the proceeding we describe the assumptions on the individual utility function of a generic agent $i$ belonging to group $G$.

\subsection{The individual utility function}

The individual utility function of agent $i$ belonging to group $G$ depends on the amount of calories she intakes through food consumption $c_{i} \geq 0$ and on her body weight $w_{i}>0^{8}$. We consider the following utility function

$$
U_{i, G}\left(c_{i}, w_{i}\right)=c_{i}\left(c_{i}^{F}-\frac{c_{i}}{2}\right)-\frac{1}{2}\left(w_{i}-w_{i}^{H}\right)^{2}-\frac{\beta}{2}\left(w_{i}-w^{G}\right)^{2} .
$$

The first term represents utility from food consumption. The parameter $c_{i}^{F}>0$ represents the individual satiation point (where $F$ stands for food) beyond which the marginal pleasure from food is negative ${ }^{9}$. We say that the agent $i$ is undereating (or equivalently on a diet) if she eats less that her satiation level, and she is overeating when she overcomes it. The former case occurs when $c_{i}<c_{i}^{F}$ and the latter when $c_{i}>c_{i}^{F}$.

The last two terms represent the effect of body weight on individual utility. We assume that this occurs through two channels: health and social desirability.

The first effect refers to the health consequences of being either overweight or underweight. According to the WHO guidelines $(1995 ; 2000 ; 2004)$ this occurs when a person's body mass index is higher than 25 or lower than 18.5, respectively. When the individual BMI is between 18.5 and 25 , a person is considered to have a normal weight 10 Within this range of values we assume that there exists for each agent $i$ a BMI that maximizes the agent's health condition, and we denote the corresponding body weight as $w_{i}^{H}>0$ (where $H$ stands for healthy) The

\footnotetext{
${ }^{8}$ Instead of referring to body weight, one might refer to body mass index, which is a measure of body weight normalized by the individual's height and is the most used diagnostic tool in the study of weight problems. More precisely, the BMI is defined as the individual's body weight (expressed in kilograms) divided by her squared height (expressed in $\mathrm{m}^{2}$ ), and it depends neither on age nor on sex. It is thus clear that, if the individual height is constant, it is equivalent to referring to individual body weight or to BMI.

${ }^{9}$ Allowing for satiation seems to be a natural assumption as we are focusing on the consumption of food at the individual level, and not on the consumption of a generic bundle of goods.

${ }^{10} \mathrm{~A}$ BMI higher than 25 indicates that the person is overweight, and when BMI $\geq 30$ the person is obese. A $17 \leq \mathrm{BMI}<18.5$ indicates that the individual is mildly underweight, when $16.5 \leq \mathrm{BMI}<17$ that is moderately underweight, and severely underweight when lower than 16.5.

${ }^{11}$ See Dwyer (1996) for a discussion on the definition of healthy weight.
} 
health consequences of body weight are summarized by the disutility cost the agent suffers when her body weight $w_{i}$ is different from her healthy weight $w_{i}^{H}$ (for a similar assumption see Etilè, 2007; Lakdawalla and Philipson, 2005; Philipson and Posner, 2003).

The second effect is related to the existence of a socially desirable body weight $w^{G} \geq 0.12 \mathrm{We}$ assume that $w^{G}$ is exogenously determined and that all agents belonging to group $G$ consider $w^{G}$ to be the socially desirable body weight. Given that agents are heterogeneous, in general $w^{G}$ does not coincide with the individual healthy weight. As in Levy (2002), the specification used in (1) implies that having a body weight that is different from $w^{G}$ is costly for the agent, with the parameter $\beta \geq 0$ measuring the relevance of this cost. We interpret this as the social cost of not conforming to a given weight due to, for example, discrimination on the job or between peers.

\subsection{The determinants of individual body weight}

Agent $i$ can choose how much to consume. She knows that her body weight cannot be directly chosen, but it is endogenously determined by the balance between calorific intake and expenditure 13 Research on human nutrition shows that the largest source of calories expenditure is due to the functioning of organs and tissues, as measured by the basal metabolic rate (BMR). The BMR is the energy expenditure when a person is at rest (Mifflin et al., 1990). It is not influenced by the level of aerobic activities, and it largely depends on body weight, together with other individual characteristics such as individual metabolism (Broeder et al., 1992; Smith et al., 1997). As in Levy (2002) and Dragone (2009), we assume that body weight changes over time according to the following law of motion:

$$
\dot{w}_{i}(t)=c_{i}(t)-\delta w_{i}(t)
$$

where $\delta>0$ is a positive parameter indicating the effect of weight on burning calories (e.g. metabolism), and $\delta w_{i}(t)$ represents the energy expenditure due to the BMR ${ }^{14}$

\subsection{The individual problem}

The goal of agent $i$ belonging to group $G$ is to choose the amount of food consumption that maximizes her utility. Given an infinite time-horizon and a discount rate $\rho>0$, the agent's

\footnotetext{
${ }^{12}$ The provocative sentence "A woman can never be too rich or too thin", attributed to Wallis Simpson, Duchess of Windsor, corresponds to the case where the ideal body weight is zero.

${ }^{13}$ If body weight could be chosen by an individual, then the determination of the individual body weight would only depend on the individual preferences.

${ }^{14}$ One might also include in the analysis the choice of physical activity, which includes on-the-job and off-the-job activity. Though it is a relevant source of calories expenditure, it turns out that it plays a minor role with respect to the BMR. In the Appendix we show that extending the analysis to include physical exercise does not change the qualitative properties of the solution.
} 
intertemporal problem can be formally written as follows

$$
\begin{aligned}
\max _{\left\{c_{i}(t)\right\}} \int_{0}^{\infty} e^{-\rho t}\left[c_{i}(t)\left(c_{i}^{F}-\frac{c_{i}(t)}{2}\right)\right. & \left.-\frac{\left(w_{i}(t)-w_{i}^{H}\right)^{2}}{2}-\beta \frac{\left(w_{i}(t)-w^{G}\right)^{2}}{2}\right] d t \\
\text { subject to } \dot{w}_{i}(t) & =c_{i}(t)-\delta w_{i}(t) \\
w_{i}(0) & =w_{i 0} .
\end{aligned}
$$

where $w_{i 0}$ is the individual body weight at time zero.

Before solving the dynamic problem, consider what would happen if the agent did not take into account how food consumption affects her body weight, i.e. if she ignored (4). In such a case, it would always be optimal to consume up to satiation for all possible body weights, i.e. agent $i$ would choose $c_{i}(t)=c_{i}^{F}$ at all $t$. The optimal consumption of this agent does not change over time, but her body weight clearly changes, according to (4), until the steady state weight $w_{i}=c_{i}^{F} / \delta$ is reached. The value $w_{i}^{F}:=c_{i}^{F} / \delta$ is to be interpreted as the body weight that an agent would reach if she always ate up to satiation, and it allows us to distinguish between two possible cases. If $w_{i}^{F}>w_{i}^{H}$, we say that the agent tends to be overweight, and we say that the agent tends to be underweight otherwise.

When moving to the dynamic problem, it is clear that choosing to eat up to satiation in general does not maximize the intertemporal discounted utility of the agent. This is apparent from the following proposition, where we characterize the solution of the intertemporal problem once the agent $i$ takes into account all factors that influence her utility, including the endogenous consequences of her eating behavior for her body weight.

Let $A=1+\beta+\delta(\delta+\rho) ; B=-\rho+\sqrt{4(1+\beta)+(2 \delta+\rho)^{2}} ; w_{i}^{W}=w_{i}^{H}-\frac{\delta(\delta+\rho)}{\beta}\left(w_{i}^{F}-w_{i}^{H}\right)$ and $w_{i}^{C}=w_{i}^{F}+\frac{1}{\beta}\left(w_{i}^{F}-w_{i}^{H}\right)$; the following then applies.

Proposition 1 Given the intertemporal problem (3)-(5):

1. there exists a unique steady state level of food consumption and body weight

$$
\begin{aligned}
c_{i}^{*} & =\delta \frac{w_{i}^{H}+\delta(\delta+\rho) w_{i}^{F}+\beta w_{i}^{G}}{A}, \\
w_{i}^{*} & =\frac{w_{i}^{H}+\delta(\delta+\rho) w_{i}^{F}+\beta w_{i}^{G}}{A} ;
\end{aligned}
$$

2. the steady state has saddle point stability;

3. in steady state, the agent is underweight if $w^{G}>w_{i}^{W}$ and overeating results if $w^{G}>w_{i}^{C}$;

4. along the optimal path leading to the steady state, food consumption is given by the following decreasing function of body weight

$$
c_{i}=\frac{B}{2 \delta} c_{i}^{*}+\left(\delta-\frac{B}{2}\right) w_{i} .
$$


Proof. The current-value Hamiltonian function associated with the problem is (omitting the time index and the individual index $i$ )

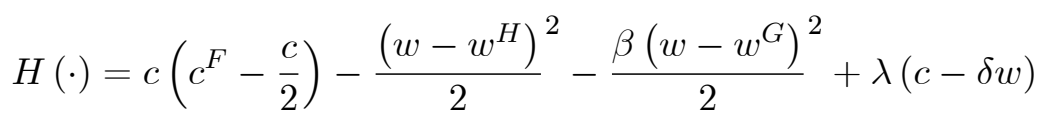

where $\lambda$ is the relevant costate variable. The necessary and sufficient conditions are:

$$
\begin{gathered}
\frac{\partial H}{\partial c}=0 \Leftrightarrow c^{F}-c=-\lambda \\
\dot{\lambda}=\rho \lambda-\frac{\partial H}{\partial w}=(\rho+\delta) \lambda+\left(w-w^{H}\right)+\beta\left(w-w^{G}\right) \\
\dot{w}=c-\delta w
\end{gathered}
$$

with the relevant transversality condition being $\lim _{t \rightarrow \infty} e^{-\rho t} \lambda(t) w(t)=0$. Differentiating 10 with respect to time and substituting (11)-(10), we can rewrite the above conditions as a linear dynamic system that depends on consumption and weight, obtaining:

$$
\begin{aligned}
\dot{c} & =\left(c-c^{F}\right)(\delta+\rho)+(1+\beta) w-w^{H}-w^{G} \\
\dot{w} & =c-\delta w .
\end{aligned}
$$

The steady state results from $\dot{c}=\dot{w}=0$. As the trace and the determinant of the $2 \times 2$ Jacobian matrix

$$
J=\left[\begin{array}{cc}
\delta+\rho & 1+\beta \\
1 & -\delta
\end{array}\right]
$$

are, respectively, positive and negative, the steady state has saddle point stability. To check that (8) the optimal trajectory leading to the steady state is indeed linear, take the ratio between (13) and (14),

$$
\frac{d c}{d w}=\frac{\left(c-c^{F}\right)(\delta+\rho)+\left(w-w^{H}\right)+\beta\left(w-w^{G}\right)}{c-\delta w},
$$

and substitute. As the slope of $(8)$ is $\delta-\frac{B}{2}<0$, the optimal path of food consumption crosses the locus $\dot{w}=0$ from above, guaranteeing that (8) is indeed the stable saddle path. To verify the conditions under which being overweight and overeating occurs take, respectively, the difference $w^{*}-w_{i}^{H}$ and $c^{*}-c_{i}^{F}$, and rearrange.

Proposition 1 shows that a forward looking agent can do better than just eating up to satiation. It is optimal to adapt the amount of food consumption to the current body weight (see Eq. 8). More precisely, it is optimal to limit food consumption when body weight is high and, conversely, to consume much (eventually beyond satiation) when body weight is too low. When the food intake follows the trajectory indicated by (8), body weight will converge to the steady state, where the choice of food consumption is such that the marginal utility of food is 
equal to the marginal disutility of weight (see equation 13). Note that both the steady state food consumption and body weight are positive functions of the healthy weight, of the body weight associated with food satiation and of the ideal body weight.

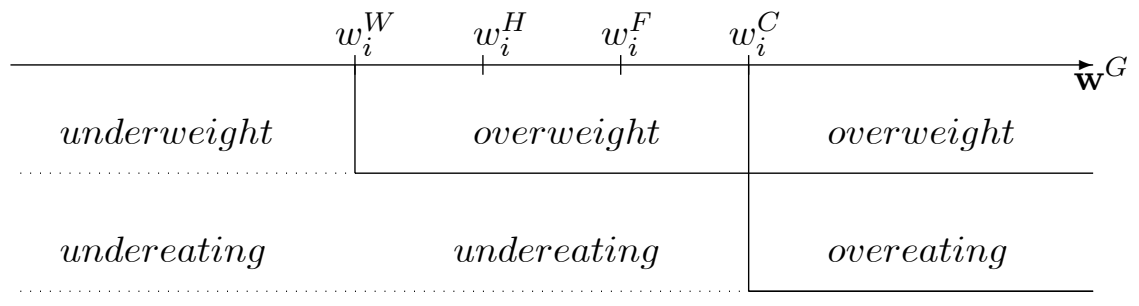

Figure 1: Possible steady states for an agent that tends to be overweight

To appreciate the implication of Proposition 1, Figure 1 shows what happens for the case where sticking to the satiating choice of food consumption would yield the agent to be overweight. In such a case $w_{i}^{W}<w_{i}^{C}$ and three cases can occur. If $w^{G}<w_{i}^{W}$, agent $i$ will be underweight and undereating; if $w^{G} \in\left(w_{i}^{W}, w_{i}^{C}\right)$ agent $i$ will be overweight and undereating; and if $w^{G}>w_{i}^{W}$ agent $i$ will be overweight and overeating. Note that the distance between $w_{i}^{W}$ and $w_{i}^{C}$ increases as $\beta$ decreases. If there were no social pressure $(\beta=0)$ only the steady state associated with being overweight and undereating could arise. If instead the satiating choice yielded to be underweight, $w_{i}^{F}<w_{i}^{H}$, and $\beta>0$, three possible steady states could arise, namely (i) a condition of being underweight and undereating, (ii) a condition of being underweight and overeating, and (iii) a condition of being overweight and overeating. Analogously to the previous case, when $\beta=0$, only the steady state associated with being underweight and overeating can be reached. Overall, this means that, depending on the individual characteristics of agent $i$ and on the ideal body weight, a variety of steady states can arise:

1. overweight and undereating;

2. overweight and overeating;

3. underweight and overeating;

4. underweight and undereating.

All steady states above can be produced within the same theoretical framework and they are consistent with the empirical observation on the eating behavior of people and the body weight they reach. The first category corresponds to those that constrain their food consumption in order to avoid becoming more overweight (i.e. fat people that stay on a diet). The second category results when the ideal weight $w^{G}$ is very high, i.e. $w^{G}>\max \left\{w_{i}^{C}, w_{i}^{W}\right\}$. Sumo wrestlers 
and body builders tend to reach this condition. The third case is typical of people that have a very fast metabolism (i.e. $\delta$ is high) and must compensate for it by overeating. The fourth category occurs when the ideal weight is very low, i.e. $w^{G}<\min \left\{w_{i}^{C}, w_{i}^{W}\right\}$. People falling into this category are thin, and yet they restrain their food consumption.

\section{Is increasing the ideal body weight desirable?}

All policies mentioned in section 2 observe that the current ideal body weight is below the average body weight of the population, and that it would be desirable to increase it. In this section we show that the desirability of increasing the ideal body weight critically depends on what the new ideal body weight is and on who will be affected by this change. In order to do so, we first determine the ideal body weight that maximizes average utility and the ideal body weight that minimizes average health losses. Then, we use these two theoretical findings to evaluate the Spanish and German proposal, according to which the ideal body weight should resemble the population biometrics.

\subsection{Optimal ideal body weight}

In the previous section we have considered the optimal behavior of a generic agent $i$ belonging to group $G$. We now focus on the whole group, assuming that all its members choose their food intake according to Proposition 1.

For all agents in $G$, the ideal body weight is the same. They are however heterogeneous in their individual healthy weights $w_{i}^{H}$ and in their satiation levels $c_{i}^{F}{ }^{15}$ Let $f_{G}\left(w^{H}, c^{F}\right)$ be the joint density function of the healthy weights $w_{i}^{H}$ and of the satiation levels $c_{i}^{F}$ of the agents belonging to group $G$. This allows us to write the average utility of group $G$ as

$$
\iint U_{i, G}\left(c_{i}, w_{i}\right) f_{G}\left(w^{H}, c^{F}\right) d w^{H} d c^{F}
$$

Define $\bar{w}^{H}$ and $\bar{c}^{F}$ as the average healthy weight and the average satiation level in group $G$, respectively. By analogy with the individual case, we define $\bar{w}^{F}=\bar{c}^{F} / \delta$ as the body weight associated to satiation and we say that the group tends to be overweight if $\bar{w}^{F}>\bar{w}^{H}$, and that it tends to be underweight otherwise.

Let $C=1 /\left\{\beta\left(1+\delta^{2}\right)+[1+\delta(\delta+\rho)]^{2}\right\}$, then the following Lemma applies:

\footnotetext{
${ }^{15}$ All the following statements are made at the group level. This implies that, given that agents are heterogeneous, it is possible that policies that are, say, welfare improving at the group level, decrease welfare for some agent. This does not affect the statements as long as the welfare losses are more than compensated for by the welfare gains of other agents in the group.
} 
Lemma 1 Given the joint density function $f_{G}\left(w^{H}, c^{F}\right)$ of the healthy weights $w_{i}^{H}$ and of the satiation levels $c_{i}^{F}$ :

1. the ideal body weight that maximizes the average steady state utility of group $G$ is

$$
w^{G \prime}=[1+\beta+\delta(\delta+2 \rho)] C \bar{w}^{H}+\delta^{2}\left[1+\beta+(\delta+\rho)^{2}\right] C \bar{w}^{F}
$$

2. the ideal body weight $w^{G \prime}$ is between the average healthy weight and the body weight associated to satiation $\bar{w}^{F}$;

3. when $w^{G}=w^{G \prime}$, on average the group will reach a steady state associated with

(a) undereating and being overweight if $\bar{w}^{F}>\bar{w}^{H}$, and

(b) overeating and being underweight if $\bar{w}^{F}<\bar{w}^{H}$.

Proof. Consider the following problem

$$
\max _{w^{G}} \iint U_{i, G}\left(w_{i}^{*}, c_{i}^{*}\right) f_{G}\left(w^{H}, c^{F}\right) d w^{H} d c^{F} .
$$

Taking the derivative with respect to $w^{G}$ yields

$$
\begin{gathered}
\iint\left\{\frac{\delta\left[(1+\beta)^{2}+(\delta+\rho)^{2}\right]}{A^{2}} c_{i}^{F}+\frac{1+\beta+\delta(\delta+2 \rho)}{A^{2}} w_{i}^{H}+\right. \\
\left.-\frac{\delta^{2} \rho^{2}+\left(\delta^{2}+1\right)[1+\beta+\delta(\delta+2 \rho)]}{A^{2}} w^{G}\right\} f_{G}\left(w^{H}, c^{F}\right) d w^{H} d c^{F}=0 .
\end{gathered}
$$

Exploiting the additive separability of the foc, it can be simplified as follows

$$
\begin{gathered}
\delta\left[1+\beta+(\delta+\rho)^{2}\right] \bar{c}^{F}+[1+\beta+\delta(\delta+2 \rho)] \bar{w}^{H}+ \\
-\left\{\delta^{2}\left(\rho^{2}+1\right)+[1+\beta+\delta(\delta+2 \rho)]\right\} w^{G}=0 .
\end{gathered}
$$

Solving with respect to $w^{G}$ yields the ideal body weight that maximizes the average utility of $G$. Substituting $w^{G \prime}$ in the steady state food consumption and body weight of the representative consumer, i.e. an agent whose satiation level and healthy weight is precisely $\bar{c}^{F}$ and $\bar{w}^{H}$, yields the last result in the Lemma.

The Lemma above implies that, under the optimal ideal body weight $w^{G}=w^{G \prime}$, group $G$ would not reach a steady state associated with being underweight and undereating, nor with being overweight and overeating. In other words, these are dominated outcomes that the policy maker would eliminate if it could optimally set the ideal body weight.

We now consider what ideal body weight would maximize the average steady state health of group $G$, which is equivalent to the solution of the following problem

$$
\min _{w^{G}} \iint\left(w_{i}^{*}-w_{i}^{H}\right)^{2} f_{G}\left(w^{H}, c^{F}\right) d w^{H} d c^{F} .
$$


Lemma 2 Given the joint density function $f_{G}\left(w^{H}, c^{F}\right)$ of the healthy weights $w_{i}^{H}$ and of the satiation levels $c_{i}^{F}$ :

1. The ideal body weight that maximizes the average steady state health of group $G$ is

$$
\bar{w}^{W}=\bar{w}^{H}-\frac{\delta(\delta+\rho)}{\beta}\left(\bar{w}^{F}-\bar{w}^{H}\right)
$$

2. the body weight $\bar{w}^{W}$ is less than the average healthy weight if the group tends towards being overweight, and it is higher if the group tends towards being underweight;

3. when $w^{G}=\bar{w}^{W}$, on average the steady state will not be associated with being overweight, nor being underweight. The steady state is characterised by

(a) undereating if $\bar{w}^{F}>\bar{w}^{H}$, and

(b) overeating if $\bar{w}^{F}<\bar{w}^{H}$.

Proof. See the previous proof, replacing the average utility function with the average loss function.

\subsection{Should the healthy weight be the ideal body weight?}

After presenting the individual model and determining the ideal body weights that maximize the average utility and health of a target group $G$, we can now focus on the case that is the major source of concern for the governments that have promoted actions against the "slimming mania". It has been suggested that a suitable ideal body weight should be the healthy weight. We now show how such an intervention would affect the eating behavior of the target group, and we assess whether it is welfare and health improving.

More specifically, we restrict our attention to women, and consider the case where there is a tendency towards being overweight and where the ideal body weight the target group refers to is lower than its average healthy weight, i.e. the case where $\bar{w}^{F}>\bar{w}^{H}>w^{G}$. The former condition seems to be consistent with the evidence that, in Western industrialized countries, obesity is increasing (Acs and Zoltan, 2007, among others). The latter is supported by the evidence that the majority of women are exposed to an ideal weight lower than their healthy body weight 16 .

From Fig. 1 one can see that the scenario under examination induces people to restrain average food consumption. Concerning the body weight that is reached in steady state, two cases can occur. If the ideal body weight is very low, i.e. $\bar{w}^{W}>w^{G}$, the agents will be on average underweight, otherwise (if $\bar{w}^{H}>w^{G}>\bar{w}^{W}$ ) the agents will be on average overweight.

According to Proposition 1, it is easy to observe that, when the ideal body weight is $\bar{w}^{H}$, the (new) steady state is characterized by being overweight and undereating. This means that the

\footnotetext{
${ }^{16}$ Fashion models and celebrities are thinner than $98 \%$ of American women (Smolak, 1996).
} 
steady state characterized by underweight and undereating could not emerge if the ideal body weight were the average body weight of the target group. The following Propositions state what are the welfare and health consequences of increasing the ideal body weight up to the average healthy weight of the target group.

Proposition 2 When the target group tends towards being overweight, increasing the current ideal body weight to the average healthy weight is welfare improving.

Proof. When the optimal ideal weight $w^{G \prime}$ is higher than the current ideal weight, increasing the latter (eventually until $w^{G \prime}$ ) is welfare improving. According to Lemma 1, when $\bar{w}^{F}>\bar{w}^{H}$, then $\bar{w}^{F}>w^{G \prime}>\bar{w}^{H}$, i.e. the optimal body weight is higher than the average healthy weight. The current ideal weight $w^{G}$ is, instead, lower than $\bar{w}^{H}$. Accordingly, increasing the ideal body weight up to the average healthy weight increases the average utility of the target group.

The Proposition shows that the above mentioned policies are well-founded and that aiming at an ideal body weight that resembles the average healthy weight of the target group increases the average utility of the group. One might wonder whether this policy also improves its health condition. The following Proposition addresses this issue.

Proposition 3 When the target group tends towards being overweight, increasing the current ideal body weight to the average healthy weight is health improving if the current ideal body weight is very low.

Proof. According to Lemma 2, when $\bar{w}^{F}>\bar{w}^{H}$, then $\bar{w}^{W}$ is lower than $\bar{w}^{H}$. If $w^{G}$ is lower than $\bar{w}^{W}$ (which leads the group to be underweight), increasing the ideal body weight is health increasing until $\bar{w}^{W}$. Let $\tilde{w}^{G}=\bar{w}^{H}-\frac{2 \delta(\delta+\rho)}{\beta}\left(\bar{w}^{F}-\bar{w}^{H}\right)$ be the threshold ideal weight that induces the target group to have a health condition identical to the one that would be reached when $\bar{w}^{H}=w^{G}$. If $w^{G}<\tilde{w}^{G}$, which means that the current ideal weight is very low, setting $\bar{w}^{H}=w^{G}$ is strictly health improving; if $\tilde{w}^{G}<w^{G}<\bar{w}^{H}$ it is strictly health worsening.

The above Proposition shows that increasing the ideal body weight up to the average healthy weight critically depends on the current ideal body weight. Accordingly, if the current ideal body weight is very low, an ideal body weight equal to the average body weight is unambiguously health improving. The current trend for "size zero" and the rising popularity of visibly underweight models and celebrities suggest that this might indeed be the relevant case (Ahern et al., 2008). If the current ideal weight were not so low, instead, increasing it up to the average healthy weight of the group would not improve the health condition. This might occur for two reasons. If $w^{G}>\bar{w}^{W}$, the target group is already overweight and it would become even more overweight. If instead $\tilde{w}^{G}<w^{G}<\bar{w}^{W}$, the group passes from being underweight to becoming overweight. This condition is not health improving because in the original scenario the group would be closer to the healthy weight than under the new scenario. It is however important to 
stress that, even if the average health condition worsens, this is compensated for by the increased utility from food consumption and the reduced social pressure (Proposition 3). Increasing the body weight from $w^{G}$ to $\bar{w}^{H}$ is therefore unambiguously welfare improving and desirable for the policy maker whenever the current ideal body weight is lower than the average healthy weight of the target group.

\section{Conclusions}

Inspired by the policies implemented by some European government to counteract unhealthy eating behavior, the present paper shows how policy actions that modify the ideal body weight affect individual eating choices and body weight. We have proposed a model where the utility of a forward-looking agent depends on (i) food consumption, (ii) the health condition, and (iii) the conformity of body weight to an ideal weight. The agent is aware of how food consumption affects body weight, and she explicitly takes this information into account when choosing how much to eat. We have shown that both a condition of being overweight and a condition of being underweight can result, and that it can be optimal to be on a diet despite being underweight, or to binge despite being overweight. These outcomes, which are often described in the psychological literature as forms of unhealthy eating, result from a rational intertemporal maximizing process of a perfectly informed agent, and are not due to bounded rationality, lack of information or self-control, or desire for self-destruction.

The model we propose can be used for evaluating the policies mentioned. Focusing on the specific proposal contained in the agreements, we study the consequences of having the healthy weight as the ideal body weight. We show that, given the current tendency towards obesity and the existence of thin ideal weights, such a policy is welfare improving, but it might have adverse effects on health if it induces people to become too overweight.

The model is a starting point for studying eating behavior under social pressure. Interesting extensions include introducing the role of peers in the definition of the ideal body weight, and allowing agents to choose their ideal body weight among multiple available ideal weights. Due to the novelty of the policies considered (the first agreement was signed in 2006), an empirical assessment does not exist. Collecting data and running a program to monitor the effects of these policies would then be a challenging development of this work. This is left for future research. 


\section{Appendix}

\subsection{Physical effort as an additional choice variable}

Consider the case where the agent can choose both the amount of food consumption $c_{i}$ and the amount of physical effort $e_{i} \geq 0$. We now show that, under this set up, the optimal solutions and steady state share the same properties illustrated in Section 3. Let the individual utility function $U_{i, G}\left(c_{i}, w_{i}, e_{i}\right)$ be additively separable and strictly concave with respect to all its arguments. To account for the fact that exerting physical effort burns calories, we consider the following law of motion for body weight

$$
\dot{w}_{i}(t)=c_{i}(t)-\delta w_{i}(t)-\varepsilon e_{i}(t)
$$

with $\varepsilon \geq 0$. The current-value Hamiltonian is (omitting the arguments and the time index to simplify the notation)

$$
H(\cdot)=U(c, w, e)+m(c-\delta w-\varepsilon e),
$$

with $m$ being the relevant costate. The necessary conditions for an internal solution are

$$
\begin{aligned}
H_{c}(\cdot) & =U_{c}(\cdot)+m=0 \\
H_{e}(\cdot) & =U_{e}(\cdot)-m \varepsilon=0 \\
\dot{m} & =(\rho+\delta) m-U_{w} \\
\dot{w} & =c-\delta w-\varepsilon e
\end{aligned}
$$

The additional insight with respect to the basic model concerns the optimal choice of physical effort $e$. From (21) and (22) one obtains the following optimality condition:

$$
\frac{U_{c}(\cdot)}{U_{e}(\cdot)}=-\frac{1}{\varepsilon}
$$

The above equation means that, irrespective of the shadow value of weight $m$, the optimal choice of consumption and effort depends on the marginal rate of substitution between food consumption and physical exercise. As $\varepsilon$ is non negative, both along the transition path and in steady state it is optimal to choose $c$ and $e$ so that the marginal utility of food consumption and the marginal utility of physical exercise have different signs. As an example, consider the case where the utility from physical effort is $\alpha e_{i}-\gamma e_{i}^{2} / 2$, with $\alpha, \gamma \geq 0$. The first term represents the benefit of physical exercise and the second term its disutility cost. Then the necessary conditions are

$$
\begin{aligned}
c-c^{F} & =m \\
\alpha-\gamma e & =\varepsilon m \\
\dot{m} & =(\rho+\delta) m+(\beta+1) w-w^{H}-\beta w^{G} \\
\dot{w} & =c-\delta w-\varepsilon e
\end{aligned}
$$


which implies the following linear relation between optimal consumption and effort

$$
e=\frac{\alpha}{\gamma}+\frac{\varepsilon}{\gamma}\left(c^{F}-c\right)
$$

The above equation has two implications. First, food consumption and physical effort are substitutes, which implies that, with respect to the benchmark model, the agent can eat more, everything else equal, if she exerts more effort. Second, we can solve the problem by focusing on body weight and consumption only, as information on the optimal effort can be simply derived by (30). Differentiating with respect to time (26), substituting the value of $e$ from (27) and the

value of $m$ from (26), the dynamics of food consumption is equivalent to (13), which implies the same asymptotic stability of the benchmark model. Let $F=(1+\beta) \varepsilon^{2}+\gamma[1+\beta+\delta(\delta+\rho)]$, then the steady state is

$$
\begin{aligned}
c^{* *} & =\frac{1}{F}\left\{\alpha \varepsilon(1+\beta)+\left[(1+\beta) \varepsilon^{2}+\gamma \delta(\delta+\rho)\right] c^{F}+\delta \gamma\left(\beta w^{G}+w^{H}\right)\right\} \\
e^{* *} & =\frac{1}{F}\left\{\alpha[1+\beta+\delta(\delta+\rho)]+\varepsilon\left[(1+\beta) c^{F}-\delta\left(\beta w^{G}+w^{H}\right)\right]\right\} \\
w^{* *} & =\frac{1}{F}\left[\left(\gamma c^{F}-\alpha \varepsilon\right)(\delta+\rho)+\left(\varepsilon^{2}+\gamma\right)\left(\beta w^{G}+w^{G}\right)\right] .
\end{aligned}
$$

It can be shown that, as in the benchmark model, both an overweight and an underweight steady state can emerge.

\section{References}

[1] Acs, Z. J., Lyles, A., eds., 2007. Obesity, business and public policy. Elgar, Cheltenham, U.K./Northampton, MA.

[2] Ahern, A. L., Bennett, K. M., Hetherington, M. M., 2008. Internalization of the ultra-thin ideal: positive implicit associations with underweight fashion models are associated with drive for thinness in young women. Eating Disorders 16, 294-307.

[3] Akerlof, G., Kranton, R., 2000. Economics and identity. Quarterly Journal of Economics $115,715-753$.

[4] Armstrong, L., 2006. Thin argument does a fat lot of good, The Times, Sept. 23. Available at http://www.timesonline.co.uk/tol/news/uk/article1074455.ece

[5] Bicchieri, C., 2006. The grammar of society. Cambridge University Press.

[6] Broeder C. E., Burrhus K. A., Svanevik L. S., Wilmore, J. H., 1992. The effects of aerobic fitness on resting metabolic rate, American Journal of Clinical Nutrition 55, 795-801.

[7] Cawley, J., 2004. The impact of obesity on wages. Journal of Human Resources 39, 451-474. 
[8] Downs, J. S., Loewenstein, G., Wisdom, J., 2009. Strategies for Promoting Healthier Food Choices. American Economic Review 99, 159-164.

[9] Dragone, D., 2009. A rational eating model of binges, diets and obesity. Journal of Health Economics 28, 799-804.

[10] Dwyer, J., 1996. Policy and Healthy Weight. Preventive Medicine 25, 30-33.

[11] Elster, J., 1989. Social norms and economic theory. Journal of Economic Perspectives 3, 99-117.

[12] Etilé, F., 2007. Social norms, ideal body weight and food attitudes. Health Economics 16, 945-966.

[13] Fallon, A., 1990. Culture in the mirror: Sociocultural determinants of body image. In: Cash, T., Pruzusky,T. (Eds.). Body images: Development deviance and change. New York: Guilford, 80-109.

[14] Fehr, E., Gächter, S., 2000. Fairness and retaliation: The economics of reciprocity. Journal of Economic Perspectives 14, 159-181.

[15] Garner, D. M., Garfinkel, P. E., Schwartz D., Thompson, M., 1980. Cultural expectations of thinness in women. Psychological Reports 47, 483-491.

[16] Garner, D. M., Garfinkel, P. E., 2009. Socio-cultural factors in the development of anorexia nervosa. Psychological Medicine 10, 647-656.

[17] Hamermesh, D. S., Biddle, J. E., 1994. Beauty and the labor market. American Economic Review 84, 1174-1194.

[18] Hemphill, C. S., Suk, J., 2009.The law, culture, and economics of fashion. Stanford Law Review 61, 1147-1199.

[19] Kilbourne, J., 1994. Still killing us softly: Advertising and the obsession with thinness. In Fallon, P., Katzman, T., Wooley, S. C. (Eds.), Feminist perspectives on eating disorders. New York: The Guilford Press, 395-418.

[20] Lakdawalla, D., Philipson, T. J., Bhattacharya, J., 2005. Welfare-enhancing technological change and the growth of obesity. American Economic Review 95, 253-257.

[21] Levy, A., 2002. Rational eating: can it lead to overweightness or underweightness? Journal of Health Economics 21, 887-899. 
[22] Mifflin, M. D., St Jeor, S.T., Hill L.A., Scott B. J., Daugherty S. A., Koh Y. O., 1990. A new predictive equation for resting energy expenditure in healthy individuals. American Journal of Clinical Nutrition 51, 241-247.

[23] Morris, S., 2006. Body mass index and occupational attainment. Journal of Health Economics 25, 347-364.

[24] Philipson, T. J., Posner, R. A., 2003. The long-run growth in obesity as a function of technological change. Perspectives in Biology and Medicine 46, 87-107.

[25] Philipson, T. J., Posner, R. A., 2008. Is the obesity epidemic a public health problem? A review of Zoltan J. Acs and Alan Lyles's obesity, business and public policy. Journal of Economic Literature 46, 974-982.

[26] Puhl, R. M., Heuer, C. A., 2009. The stigma of obesity: a review and update. Obesity 17, 941-964.

[27] Raustiala, K., Sprigman C. J., 2009. The piracy paradox revisited. Stanford Law Review $61,1201-1225$.

[28] Smith D. A., Dollman J., Withers R. T., Brinkman M., Keeves J. P., Clark, D. G., 1997. Relationship between maximum aerobic power and resting metabolic rate in young adult women. Journal of Applied Physiology 82, 156-163.

[29] Smolak, L., 1996. Methodological implications of a developmental psychopathology approach to the study of eating problems. In: Smolak, L., Levine M.P. (Eds). The developmental psychopathology of eating disorders: Implications for research, prevention, and treatment, Lawrence Erlbaum Associates, Inc., Hillsdale, NJ, England, 31-55.

[30] Strahan, E.J., Wilson, A.E., Cressman, K. E., Buote, V. M., 2006. Comparing to perfection: How cultural norms for appearance affect social comparisons and self-image. Body Image, $211-227$.

[31] Swami, V. (2006). The influence of body weight and shape in determining female and male physical attractiveness. In: Kindes, M. V., (Ed). Body image: new research. Nova Biomedical Books, 35-61.

[32] Turner, J., 2006. Size 0: not the thin end of the wedge, The Times, Sept. 23. Available at http://www.timesonline.co.uk/tol/comment/columnists/janice_turner/article647974.ece

[33] Wansink, B., Just, D. R., Payne, C. R., 2009. Mindless eating and healthy heuristics for the irrational. American Economic Review 99, 165-169. 
[34] WHO, 1999. Physical status: the use and interpretation of anthropometry. Report of a WHO Expert Committee. WHO Technical Report Series 854. Geneva: World Health Organization.

[35] WHO, 2000. Obesity: preventing and managing the global epidemic. Report of a WHO Consultation. WHO Technical Report Series 894. Geneva: World Health Organization.

[36] WHO expert consultation, 2004. Appropriate body-mass index for Asian populations and its implications for policy and intervention strategies. The Lancet 363(9403), 157-163.

[37] Wolf, N., 1991. The beauty myth: How images of beauty are used against women. New York: William Morrow.

[38] Yaniv, G., 2002. Non-adherence to a low-fat diet: an economic perspective. Journal of Economic Behavior and Organization 48, 93-104.

[39] Yaniv, G., Rosin, O., Tobol, Y., 2009. Junk-food, home cooking, physical activity and obesity: the effect of the fat tax and the thin subsidy. Journal of Public Economics 93, 823-830. 